\section{Eficacia de los tipos de aislamiento utilizados en clínicas de Odontopediatría UCSG, semestre B-2019}

Efficacy of the types of isolation used in UCSG Pediatric Dentistry clinics, semester B-2019.

\section{María de Lourdes Sánchez Romero}

Egresada de la Universidad Católica de

Santiago de Guayaquil,

mariasanchez@hotmail.com

https://orcid.org/0000-0002-5939-1080

Adriana Rocio Amado Schneider

Especialista en Odontopediatría y

Ortodoncia, Docente Universidad Católica

de Santiago de Guayaquil.

adrianaamados@hotmail.com

https://orcid.org/0000-0002-3478-7505

Guayaquil - Ecuador

http://www.jah-journal.com/index.php/jah

Journal of American health

Octubre - Diciembre vol. 3. Num. 3 - 2020

Esta obra está bajo una Licencia Creative Commons

Atribución-NoComercial-CompartirIgual 4.0 Internacional.

RECIBIDO: 8 DE MAYO 2019

ACEPTADO: 18 DE NOVIEMBRE 2019

PUBLICADO: 4 DE OCTUBRE 2020

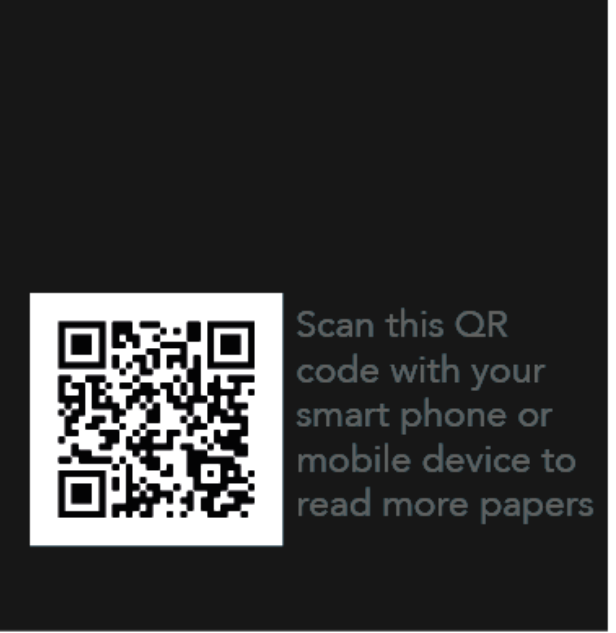

\section{RESUMEN}

Introducción: El aislamiento dental es un procedimiento clínico que previene $y$ controla fluidos salivales, generando un ambiente óptimo para su rehabilitación. Existen dos tipos de aislamiento dental: aislamiento absoluto y aislamiento relativo. Objetivo: Establecer la eficacia del tipo de aislamiento utilizado en las Clínicas de Odontopediatría UCSG, durante el semestre B-2019 por medio de una valoración observacional. Materiales y Métodos: En el presente trabajo de investigación científica se aplicó un enfoque tipo transversal, analítica y descriptiva. La muestra estuvo conformada por 103 pacientes, en edades de 5 a 11 años de género femenino y masculino, pasando por un análisis estadístico descriptivo. Resultados: Tras la evaluación clínica y estadística de las muestras alcanzadas, se consideró un porcentaje de $58 \%$ en la frecuencia del aislamiento absoluto, y en cuanto a su efectividad se obtuvo "muy buena" 55\%, mientras que el aislamiento relativo demostró una frecuencia de $42 \%$ y $2 \%$ de efectividad según el operador de ser "muy buena" la calidad de su tratamiento dental. Conclusión: Se concluye, que el tipo de aislamiento de mayor eficacia en las clínicas de odontopediatría UCSG es el aislamiento absoluto, ya que demostró confirmar sus criterios de diagnóstico para conseguir calidad en el tratamiento.

PALABRAS CLAVE: Dique de Goma, Aislamiento Relativo, Ansiedad dental, Rehabilitación oral, Odontopediatría, preferencia del paciente

\section{ABSTRACT}

Introduction: Dental isolation is a clinical procedure that prevents and controls 
salivary fluids, generating an optimal environment for rehabilitation. There are two types of dental isolation: absolute isolation and relative isolation. Objective: To establish the effectiveness of the type of isolation used in the UCSG Pediatric Dentistry Clinics during B-2019 semester, through an observational assessment. Materials and Methods: A cross-sectional, analytical, descriptive approach was applied in the present scientific research work. The sample consisted of 103 patients, between ages of 5 to 11, female and male gender, through a descriptive statistical analysis. Results: After the clinical and statistical evaluation of the sample reached, a percentage of $58 \%$ in the frequency of absolute isolation was considered, and in terms of its effectiveness, 55\% was obtained "very good", while the relative isolation showed a frequency of $42 \%$ and $2 \%$ effectiveness according to the operator of being "very good" in the quality of their dental treatment. Conclusion: It is concluded that the most effective type of isolation in UCSG pediatric dentistry clinics is absolute isolation, since it proved to confirm its diagnostic criteria to get quality treatment.

KEYWORDS: Rubber Dam, Relative Isolation, Dental Anxiety, Oral Rehabilitation, Pediatric Dentistry, Patient Preference

\section{INTRODUCCIÓN}

El aislamiento dental es un procedimiento clínico que previene y controla fluidos salivales; generando un ambiente óptimo para su rehabilitación. Existen dos tipos de aislamiento dental: aislamiento absoluto y aislamiento relativo1.

Barnum en el año de 1864 inició con aislar un molar inferior con tela de goma. A continuación, en 1882, compañía SS White dio a conocer un similar dique de goma al actual, Delous Palmer implantó múltiples grapas en el mismo año. Lamper, 1977 expuso que el desistir al uso de dique y el paciente deglute o aspira un material o instrumento, dicho descuido apenas encontrara protección legal ante un tribunal 1,3,9.

En la cavidad bucal puede presentarse diferentes razones que afecten la operatoria dental, como limitación del campo visual, acceso bucal, humedad, filtración de flora bacteriana entre otros; por lo que el uso de aislamiento es recomendado para asegurar el éxito de la operatoria dental2.

La selección del tipo de aislamiento dental está relacionada con el tipo de protección que necesitamos para el paciente y según el tipo de procedimientos dentales que vamos a realizar. El uso de aislamiento absoluto en Odontopediatría es muy importante para evitar la aspiración de instrumentos dentales, protección de tejidos blandos, y facilita el control de la lengua evitando contaminar el área a trabajar2-3.

Estudios realizados en el 2013, informaron de la tasa de éxito gracias al aislamiento absoluto fue de un 93,3\%; en comparación de los que no realizaron el aislamiento absoluto fue de un 73,6\%1-4. 
El porcentaje global del uso de aislamiento absoluto según rehabilitadores es de un 89,6\%; en comparación en los demás procedimientos dentales como los endodónticos, o los de odontología general2,4-5.

Con la presente investigación se propondrá establecer la eficacia del tipo de aislamiento utilizado en la Clínica de Odontopediatría. Contribuye a un mayor conocimiento para la correcta elección del tipo de aislamiento según diferentes parámetros que se medirán en la investigación por parte del odontólogo.

\section{MATERIALES Y MÉTODOS}

En el presente trabajo de investigación científica se aplicó un enfoque tipo transversal, analítica y descriptiva. La muestra estuvo conformada por 103 pacientes, en edades de 5 a 11 años de género femenino y masculino, los mismos quienes fueron observados en la clínica odontológica de la Universidad Católica de Santiago de Guayaquil. Tomando en cuenta los criterios de inclusión que formaron parte del estudio, pacientes atendidos en las clínicas de Odontopediatría I y Odontopediatría II, con carácter voluntario se obtuvo la autorización por medio del consentimiento informado firmado por padres o tutores, pacientes con indicación de aislamiento absoluto, o pacientes con indicación de aislamiento relativo. Se tramitó la autorización a las autoridades respectivas para ingresar a las clínicas de Odontopediatría I y Odontopediatría II durante el período 2019-2020, posteriormente se seleccionó el tamaño de la muestra según los criterios de inclusión y exclusión. Continuo a este proceso a cada paciente que fue seleccionados se les procedía a indicar a los padres o tutores los procedimientos a seguir, se les hacía la entrega del consentimiento informado para su autorización para la observación del comportamiento del niño o niña ante el tipo de aislamiento pertinente para el tratamiento a realizar, también observar la eficacia del tipo de aislamiento en el mismo, luego de recolectar los datos del paciente se observaba el nivel de ansiedad ante cada paso previo a la colocación del aislamiento tanto absoluto como relativo, el cual era medido ante la escala de imagen facial (FIS) que residía en una escala con un número consolidado de cinco caras clasificadas desde "muy feliz" a "muy triste", y ante el comportamiento del niño era proporcionado mediante la escala de Frankl que consistía en mencionar desde un Tipo 1 que resultaba ser un comportamiento "definitivamente negativo" a un tipo 4 que era un comportamiento "definitivamente positivo", luego se procedía a preguntar al operador sobre cuán a menudo usaba aquel tipo de aislamiento, que criterios tenía para usarlo y que tan eficaz le resultaba su uso, en el transcurso del tratamiento, con la ayuda de un espejo bucal se observaba si habían filtraciones de líquidos (saliva o sangre) hacia el cuadrante o pieza a trabajar, lo que me servía para constatar la eficacia de uso del aislamiento. utilizado ante cada tratamiento. Para culminar, los datos fueron resumidos en una hoja de registro, la misma que pasó por un análisis estadístico para proporcionar un resultado en base a la estadística descriptiva, mediante el uso del programa Microsoft Office Excel 2016.

\section{RESULTADOS}

La intención del estudio fue definir la frecuencia del aislamiento absoluto (dique de goma) vs aislamiento relativo del campo operatorio en procedimientos clínicos, para obtener este 
resultado se observaba o preguntaba al operador sobre que tipo de aislamiento utilizaría en su acción clínica a realizar.

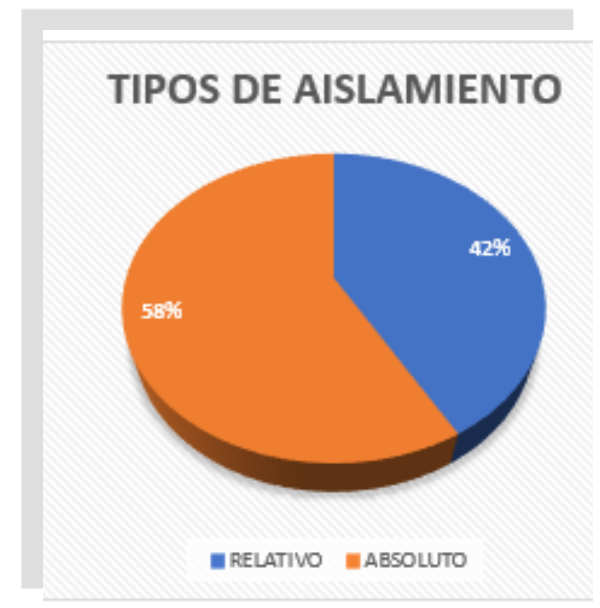

Gráfico 1. Aislamiento Absoluto $58 \%$ vs Aislamiento Relativo $42 \%$.

De acuerdo con la tabla anexa de las muestras alcanzadas se considera una mayor frecuencia en el uso del aislamiento absoluto con un $58 \%$ continuo a ello, el aislamiento relativo con un $42 \%$.

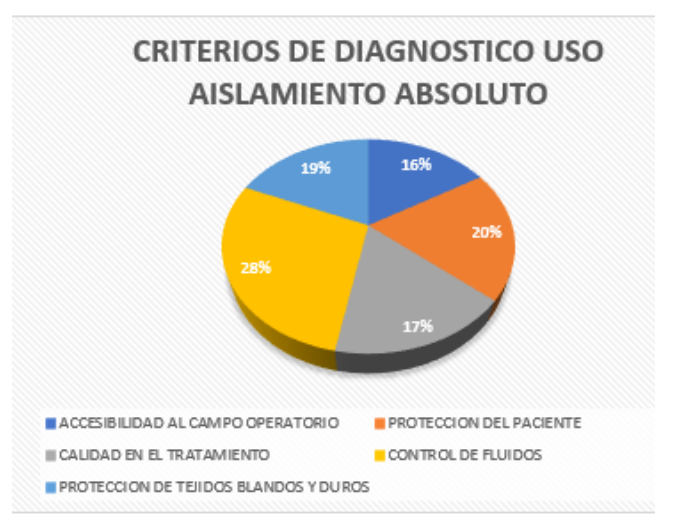

Gráfico 2. Criterios de diagnóstico para Aislamiento absoluto. 
Analizar los factores que inciden en la elección del tipo de aislamiento, según el tipo de aislamiento absoluto es usada con un $28 \%$ por criterios de control de fluidos en el campo operatorio, mientras que el aislamiento relativo con un $43 \%$ de porcentaje que usan por accesibilidad al campo operatorio.

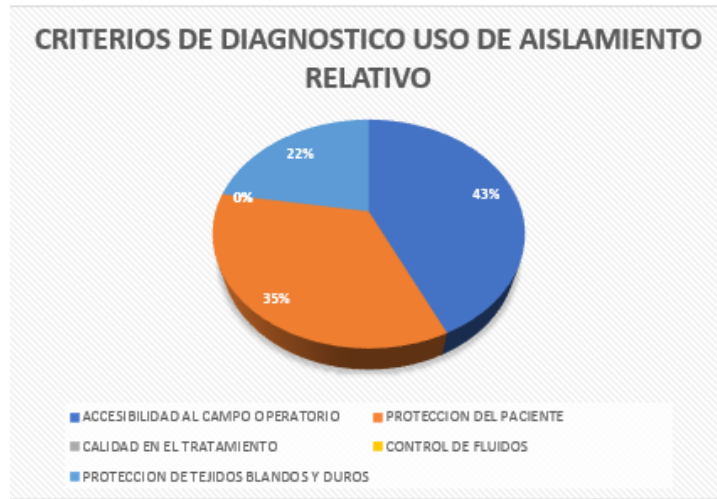

Gráfico 3. Criterio para Aislamiento relativo, presentó mayor porcentaje la accesibilidad al campo operatorio.

De acuerdo con la ansiedad del paciente en el momento del tratamiento es de $46 \%$ se encuentran "muy feliz" y un $2 \%$ "muy triste" siendo medidos por la escala de imagen facial (FIS)

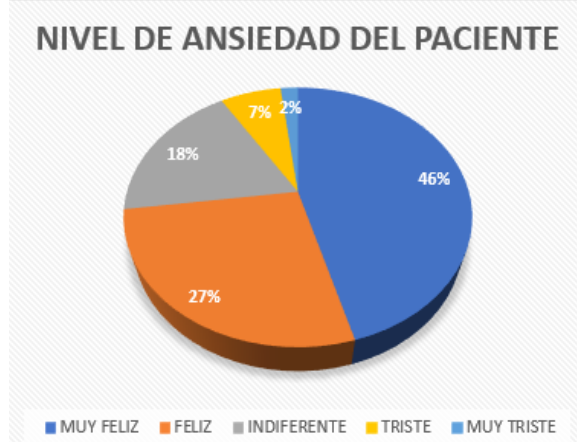

Gráfico 4. Nivel de Ansiedad del paciente

Mientras que, al relacionar el uso del aislamiento absoluto con la reducción de niveles de ansiedad del paciente, mediante la observación en la escala de imagen facial (FIS), se mostró $53 \%$ "muy bien" 


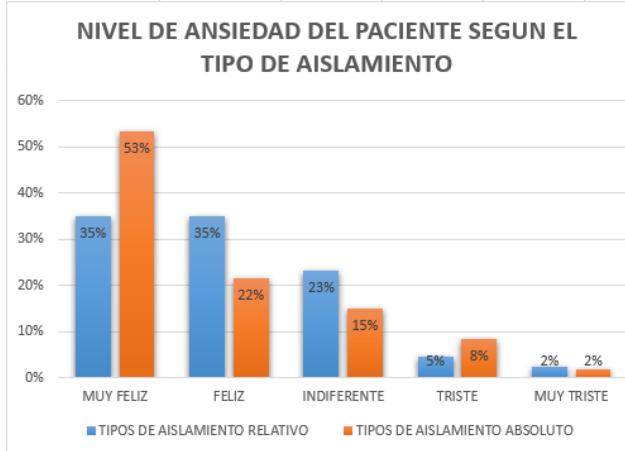

Gráfico 5. Niveles ansiedad del paciente según la relación aislamiento absoluto o relativo.

Determinar el nivel de colaboración del paciente, a la estadística el 50\% es "definitivamente positivo" y hay un $3 \%$ que a la colaboración refiere "definitivamente negativo", según la escala de Frankl.

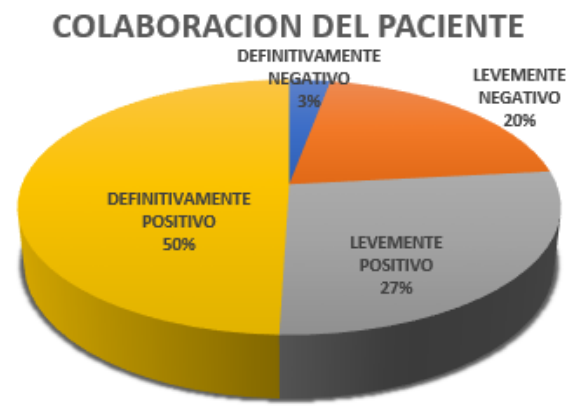

- DEFINITIVAMENTE NEGATIVO

- LEVEMENTE POSITIVO

Gráfico 6. Colaboración del paciente.

Al determinar el nivel de colaboración del paciente que interviene en la elección del tipo de aislamiento en el campo operatorio reveló que ante el aislamiento absoluto es de $50 \%$ "definitivamente positiva" y un $3 \%$ es "definitivamente negativo" según la escala de Frankl mientras tanto el aislamiento relativo con porcentaje de $37 \%$ en "definitivamente positivo" $2 \%$ es "definitivamente negativo".

\section{COLABORACION DEL PACIENTE \\ SEGUN EL TIPO DE \\ AISLAMIENTO}

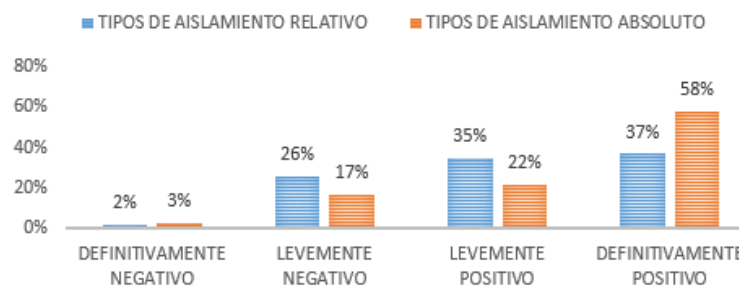

Gráfico 7. Colaboración del paciente según el tipo de aislamiento absoluto y relativo. 
Establecer la percepción del operador sobre la efectividad del tipo de aislamiento en la calidad de su tratamiento restaurador usando el aislamiento absoluto o el aislamiento relativo, mediante el cual se obtuvo 55\% en el aislamiento absoluto de ser "muy bien" su efectividad, mientras que en el caso del aislamiento relativo demuestra un $2 \%$ de efectividad según el operador de ser "muy bien" la calidad de su tratamiento restaurador según la escala de Likert.

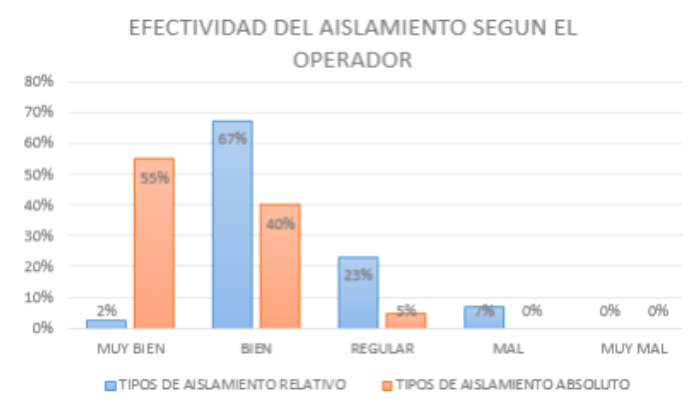

Gráfico 8. Efectividad del aislamiento según el operador.

\section{DISCUSIÓN}

Estudios reportan el porcentaje global de uso de aislamiento absoluto entre los rehabilitadores orales en un $89,6 \%$, el que fue elevado en relación con lo reportado en procedimientos endodónticos y en restauraciones por odontólogos generales. En cuanto al uso de dique de goma en egresados del pregrado se encontró un porcentaje de $57,4 \%$ entre los graduados de los últimos 14 años, en comparación con 42,6\% en los graduados de más de 14 años 2.

Feierabend \& et al, reportan los criterios para la elección de aislamiento más predominantes entre los rehabilitadores y endodoncistas fueron los de evitar el contacto con humedad, saliva y fluidos; además de mejorar la visibilidad y acceso al campo operatorio3. En el presente estudio se tuvo similares inclinaciones ya sea por la parte de restauraciones complejas, tratamientos endodónticos en niños denominados pulpotomía y con respecto a criterios de diagnóstico del operador, entre los de primera elección se menciona el control de fluidos, proteger al paciente, sus tejidos blandos y duros para de esta manera conseguir calidad en el tratamiento.

Mautz \& et al, reportan estudios realizados en Brazil donde median la prevalencia de la ansiedad dental del $16,8 \%$ en niños y niñas de 5 años. Sin embargo, otros estudios como Bezabih \& et al, reportan una ansiedad dental moderada a severa de un $74,1 \%$ en pacientes pediátricos de Etiopía y en Pakistán un $38 \%$ de prevalencia de ansiedad dental según Raja \& et al24.

Según Alcagaya, 2004 menciona que en la primera consulta se despliegan los sentimientos del paciente respecto al tratamiento odontológico y al dentista. Sanchis, 2011 menciona que la colaboración del niño es necesario llevar a cabo un tratamiento dental de calidad en la 
consulta; los factores que influyen sobre la conducta del paciente infantil son muchos y variados.

Se demostró en el presente estudio usando la Escala de Imagen Facial que el nivel de ansiedad en un 53\% era "muy feliz". Y la colaboración del paciente era de un $58 \%$ siendo "definitivamente positivo" ante el aislamiento absoluto.

Collette \& et al, evaluó el tipo de aislamiento en relación con el éxito del tratamiento midiendo el confort del paciente, y el tiempo requerido durante el tratamiento. En casos de tratamientos rehabilitadores se observó mayor éxito en casos con aislamiento absoluto y en casos como aplicación de sellantes se evidenció mayor uso de aislamiento relativo según la edad del paciente a utilizar21.

Mediante esta investigación se corrobora que el uso de aislamiento absoluto tiene mayor eficacia en los tratamientos.

\section{CONCLUSIONES}

Se concluye, que el aislamiento absoluto fue de mayor eficacia en las clínicas de odontopediatría UCSG, además que demostró confirmar los criterios de diagnóstico, para conseguir calidad en el tratamiento expuestos por Feierabend \& et al, además que el nivel de ansiedad en los niños se encuentra "muy feliz"(FIS) durante el procedimiento clínico usando aislamiento absoluto.

\section{REFERENCIAS}

1. Gómez Díaz M, Vargas Quiroga E, Pattigno Forero B, Tirado Amador L. Algunas consideraciones sobre el aislamiento absoluto. MEDISAN [Internet]. octubre de 2017 [citado 19 de mayo de 2019];21(10):3066-76. Disponible en: http://scielo.sld.cu/scielo.php?script=sci_abstract\&pid=S102930192017001000011\&lng=es\&nrm=iso\&tlng=es

2. Pineda JLG, Falla JCR, Grisales RF, Zapata SM. Uso de aislamiento absoluto con dique de goma en tratamientos restaurativos por rehabilitadores orales en el departamento de Antioquia. Rev CES Odontol [Internet]. 2018 [citado 19 de mayo de 2019];31(2):28-37. Disponible en: https://dialnet.unirioja.es/servlet/articulo?codigo=6815148

3. Feierabend S, Matt J, Klaiber B. A Comparison of Conventional and New Rubber Dam Systems in Dental Practice. Oper Dent [Internet]. mayo de 2011 [citado 26 de mayo de 2019];36(3):243-50. Disponible en: http://www.jopdentonline.org/doi/10.2341/09-283-C

4. (7) Dique de goma en odontopediatría: comodidad del paciente [Internet]. ResearchGate. [citado 12 de junio de 2019]. Disponible en: https://www.researchgate.net/publication/237642158_Dique_de_goma_en_odontopedi atria_comodidad_del_paciente 
5. Saha A, Kamatham R, Mallineni S, Nuvvula S. A cross-sectional survey on children perception of isolation methods for restorative procedures and influence of cognitive development. SRM J Res Dent Sci [Internet]. 2016 [citado 26 de mayo de 2019];7(4):219. Disponible en: http://www.srmjrds.in/text.asp?2016/7/4/219/195623

6. Varughese RE, Andrews P, Sigal MJ, Azarpazhooh A. An Assessment of Direct Restorative Material Use in Posterior Teeth by American and Canadian Pediatric Dentists: II. Rubber Dam Isolation [Internet]. 2016 [citado 26 de mayo de 2019]. Disponible en: https://www.ingentaconnect.com/content/aapd/pd/2016/00000038/00000007/art0000 9

7. Soldani F, Foley J. An assessment of rubber dam usage amongst specialists in paediatric dentistry practising within the UK. Int J Paediatr Dent [Internet]. enero de 2007 [citado 25 de mayo de 2019];17(1):50-6. Disponible en: http://doi.wiley.com/10.1111/j.1365263X.2006.00796.x

8. Cross-Sectional Evaluation of Dentists Knowledge and Awareness towards Rubber Dam Usage in Pediatric Dental Procedures: An Original Study - ProQuest [Internet]. [citado 26 de mayo de 2019]. Disponible en: https://search.proquest.com/openview/c223195c0a51416b8c8a07688e9f3700/1?pqorigsite=gscholar $\& \mathrm{cbl}=2040251$

9. Imbery T, Greene K, Carrico C. Dental Dam and Isovac Usage: Factors Influencing Dental Students' Decisions on Isolation Techniques. J Dent Educ [Internet]. 1 de abril de 2019 [citado 26 de mayo de 2019];83(4):474-82. Disponible en: http://www.jdentaled.org/content/83/4/474

10. Ahmed HMA, Cohen S, Lévy G, Steier L, Bukiet F. Rubber dam application in endodontic practice: an update on critical educational and ethical dilemmas. Aust Dent J. diciembre de 2014;59(4):457-63.

11. Hill EE. Do Dental Educators Need to Improve Their Approach to Teaching Rubber Dam Use? J Dent Educ. 2008;72(10):5.

12. Pol S, Katge F, Krishna V, Balgi P, Pradhan D. Effect of rubber dam on objective and subjective parameters of stress during dental treatment of children. Indian J Oral Health Res [Internet]. 2018 [citado 26 de mayo de 2019];4(1):16. Disponible en: http://www.ijohr.org/text.asp?2018/4/1/16/245674

13. Van Dijken JWV, Hörstedt P. Effect of the use of rubber dam versus cotton rolls on marginal adaptation of composite resin fillings to acid-etched enamel. Acta Odontol Scand 
[Internet]. enero de 1987 [citado 26 de mayo de 2019];45(5):303-8. Disponible en: http://www.tandfonline.com/doi/full/10.3109/00016358709096351

14. Alhareky MS, Mermelstein D, Finkelman M, Alhumaid J, Loo C. Efficiency and Patient Satisfaction with the Isolite System Versus Rubber Dam for Sealant Placement in Pediatric Patients [Internet]. 2014 [citado 26 de mayo de 2019]. Disponible en: https://www.ingentaconnect.com/content/aapd/pd/2014/00000036/00000005/art0000 7

15. Hernández Dávila A. Influencia de la estructura familiar en el comportamiento del paciente en la consulta odontopediátrica. 2012 [citado 26 de mayo de 2019]; Disponible en: http://cd.dgb.uanl.mx//handle/201504211/5489

16. Al P, M C, M M. Experience and Practicality of Rubber Dam Use among Undergraduate Dental Students in Fiji. J Oral Hyg Health [Internet]. 21 de abril de 2018 [citado 26 de mayo de 2019];6(2):1-5. Disponible en: https://www.omicsonline.org/open-access/experienceand-practicality-of-rubber-dam-use-among-undergraduate-dental-students-in-fiji-23320702-1000239-100695.html

17. Haruyama A, Kameyama A, Tatsuta C, Ishii K, Sugiyama T, Sugiyama S, et al. Influence of different rubber dam application on intraoral temperature and relative humidity. Bull Tokyo Dent Coll. 2014;55(1):11-7.

18. Ammann P, Kolb A, Lussi A, Seemann R. Influence of rubber dam on objective and subjective parameters of stress during dental treatment of children and adolescents - a randomized controlled clinical pilot study: Influence of rubber dam on stress parameters. Int J Paediatr Dent [Internet]. marzo de 2013 [citado 26 de mayo de 2019];23(2):110-5. Disponible en: http://doi.wiley.com/10.1111/j.1365-263X.2012.01232.x

19. Cajazeira MRR, De Sabóia TM, Maia LC. Influence of the operatory field isolation technique on tooth-colored direct dental restorations. Am J Dent. junio de 2014;27(3):155-9.

20. Arushi G, Nikkhil S, Vivek R, Noopur K. Isolation In Pediatric Dentistry. ejpmr. 2019;6(5):491-5.

21. Lyman T, Viswanathan $\mathrm{K}, \mathrm{McWhorter} \mathrm{A}$. Isolite Vs Cotton Roll Isolation in the Placement of Dental Sealants [Internet]. 2013 [citado 26 de mayo de 2019]. Disponible en: https://www.ingentaconnect.com/content/aapd/pd/2013/00000035/00000003/art0001 9

22. Khan HR, Azam S, Qureshi B. KNOWLEDGE AND ATTITUDE OF HOUSE OFFICERS REGARDING RUBBER DAM USE. Pak Oral Dent J [Internet]. 24 de mayo de 2018 [citado 26 
de mayo de 2019];38(1):97-101. Disponible en: http://www.podj.com.pk/index.php/podj/article/view/145

23. Awooda EM, Alwan MS. Knowledge, Attitudes and Practice of Rubber Dam use among dentists working in private clinics in Khartoum City. 2016;1:5.

24. Mautz-Miranda C, Fernández-Delgadillo C, Saldivia-Ojeda C, Rodríguez-Salinas C, Riquelme-Carrasco S, Linco-Olave J, et al. Prevalencia de Ansiedad Dental en Niños Atendidos en los Servicios de Salud Públicos de Valdivia, Chile. Odontoestomatología [Internet]. diciembre de 2017 [citado 26 de mayo de 2019];19(30):59-64. Disponible en: http://www.scielo.edu.uy/scielo.php?script=sci_abstract\&pid=S1688-

93392017000300059\&lng=es\&nrm=iso\&tlng=es

25. Chagoya LAM, Dyck LPE, Valle JAJ del, Gómez MD. Prevención de la ingesta de cuerpos extraños durante la consulta odontológica: reporte de caso. Rev Mex Estomatol [Internet]. 1 de enero de 2017 [citado 25 de mayo de 2019];3(2):50-60-60. Disponible en: https://www.remexesto.com/index.php/remexesto/article/view/68

26. Mirza AJ, Javaid MA, Asghar S, Siddiqui AA, Berkathullah M. Rubber Dam: Is it a Popular Method of Field Isolation among Dentists in Karachi? 2017;(22):7.

27. Innes N. Rubber dam use less stressful for children and dentists: Question: When placing fissure sealants does rubber dam compared with cotton wool rolls reduce stress and treatment times? Evid Based Dent [Internet]. junio de 2012 [citado 25 de mayo de 2019];13(2):48-48. Disponible en: http://www.nature.com/articles/6400859

28. (7) (PDF) Short communication: Influence of different isolation methods on the survival of proximal ART restorations in primary molars after two years [Internet]. ResearchGate. [citado 12 de junio de 2019]. Disponible en: https://www.researchgate.net/publication/44635424_Short_communication_Influence_ of_different_isolation_methods_on_the_survival_of_proximal_ART_restorations_in_pri mary_molars_after_two_years

29. Akbar I, Alam F, Qureshi B, Almayouf MA. THE ATTITUDE OF UNDERGRADUATE DENTAL STUDENTS TOWARDS THE USE OF RUBBER DAM. Pak Oral Dent J [Internet]. 31 de diciembre de 2017 [citado 26 de mayo de 2019];37(4):622-7. Disponible en: http://podj.com.pk/index.php/podj/article/view/33

30. Olatosi O, Nzomiwu C, Erinoso O, Oladunjoye A. Undergraduate dental students' perception, educational satisfaction, and attitude regarding the use of rubber dam. J Clin Sci [Internet]. 2018 [citado 26 de mayo de 2019];15(1):13. Disponible en: http://www.jcsjournal.org/text.asp?2018/15/1/13/226045 
31. Madarati AA. Why dentists don't use rubber dam during endodontics and how to promote its usage? BMC Oral Health [Internet]. diciembre de 2016 [citado 26 de mayo de 2019];16(1). Disponible en: http://www.biomedcentral.com/1472-6831/16/24 